\title{
Is pigmentation important for face recognition? Evidence from contrast negation
}

\author{
Richard Russell $\uparrow$, Pawan Sinha \\ E25-225, Department of Brain and Cognitive Sciences, Massachusetts Institute of Technology, \\ Cambridge, MA 02139, USA; e-mail: rrussell@fas.harvard.edu \\ Irving Biederman§, Marissa Nederhouser \\ Department of Psychology, HNB 316, Hedco Neurosciences Building (§ also Neuroscience Program), \\ University of Southern California, Los Angeles, CA 90089, USA \\ Received 28 January 2005, in revised form 7 June 2005; published online 26 April 2006
}

\begin{abstract}
It is extraordinarily difficult to recognize a face in an image with negated contrast, as in a photographic negative. The variation among faces can be partitioned into two general sources: (a) shape and (b) surface reflectance, here termed 'pigmentation'. To determine whether negation differentially affects the processing of shape or pigmentation, we made two sets of faces where the individual faces differed only in shape in one set and only in pigmentation in the other. Surprisingly, matching performance was significantly impaired by contrast negation only when the faces varied in pigmentation. This provides evidence that the perception of pigmentation, not shape, is selectively disrupted by negation and, by extension, that pigmentation contributes to the neural representation of face identity.
\end{abstract}

\section{Introduction}

Faces are particularly difficult to recognize in photographic negatives. This phenomenon was first reported in the scientific literature by Galper (1970; Galper and Hochberg 1971), and has since been studied extensively in the vision science community (Bruce and Langton 1994; Bruce and Young 1998; George et al 1999; Hayes et al 1986; Johnston et al 1992; Kemp et al 1990, 1996; Liu et al 1999; Phillips 1972; White 2001), with the belief that determining how recognition can be impaired helps us understand how it works under normal conditions. Negation reverses the contrast polarities of an image, making black areas white, light gray areas dark gray, and so forth. It is a reversible manipulation that does not remove any information from the image. Though no information is lost, our ability to use the information in the image is severely compromised. This suggests that some normally useful information is rendered unusable by negation. It cannot be the only useful information for face recognition, as negation leads to partial rather than total impairment of performance-those cues still usable after negation should also be usable under normal conditions. But whatever information is compromised by negation is likely to be among the important components of facial identity under normal conditions.

One cue that is likely disrupted by negation is the pattern of shading across a face, which is a product of the interaction between the shape of the face and the direction of lighting. Faces are normally viewed in positive contrast, with lighting from above. Both negation and lighting from below disrupt the normal patterns of shading across a face and impair recognition. Because patterns of shading can be used to estimate 3-D shape (Horn 1986; Johnston and Passmore 1994; Ramachandran 1988), abnormal patterns of shading may cause impairment in the ability to determine the 3-D surface of the face (Hill and Bruce 1996; Johnston et al 1992; Kemp et al 1996). However, faces presented with abnormal patterns of shading but veridical 3-D stereo cues are still difficult to recognize, suggesting that abnormal shading may impair 2-D

- Current address: Department of Psychology, Harvard University, 33 Kirkland Street, \#720, Cambridge, MA 02138, USA. 
pattern processing rather than 3-D processing of shapes (Liu et al 2000). The strongest evidence that abnormal shading patterns disrupt recognition comes from work with unpigmented 3-D models of faces. With images from such models, matching performance is about $10 \%$ better with positive contrast when the head model is lit from above, but is actually better with negative contrast when the lighting is from below (Liu et al 1999). When lighting was at $0^{\circ}$ elevation (front lighting) performance was about equal with positive and negative contrast, suggesting that shading cues are minimized or eliminated by this kind of lighting. However, with normally pigmented faces, performance is significantly better in positive contrast with either top or front lighting (Johnston et al 1992). This suggests that disruption in shading is only a partial explanation why faces are difficult to recognize in negative.

It has been suggested that negation disrupts the use of two other cues: 'second-order relations' [the distances between facial features (Diamond and Carey 1986), sometimes called 'configuration']; and pigmentation. Second-order relations are widely believed to play a key role in the perceptual representation of faces. Deficits in recognition performance with vertically inverted faces have been attributed to impaired extraction of second-order relations in such stimuli (Freire et al 2000; Leder and Bruce 2000; Leder et al 2001; LeGrand et al 2001). Several authors have raised the possibility that the perception of second-order relations may be impaired also for negated faces (Hole et al 1999; Kemp et al 1990; Lewis and Johnston 1997; White 2001). Second-order relations are a subset of the larger class of shape cues that can be used to differentiate faces. We can ask whether shape cues more generally are disrupted by negation. If shape cues are not disrupted by negation, it follows that the subordinate class of second-order relations is not disrupted by negation.

It has also been proposed that the processing of another cue-'pigmentation'-is disrupted by negation. We extend the meaning of pigmentation in the current article to refer to all surface reflectance properties. Most prominent among these reflectance properties are albedo (the proportion of light of all wavelengths reflected by the surface, affecting how light or dark the surface appears) and hue (differential reflectance of particular wavelengths of light results in the surface appearing to have a particular hue, such as red or blue). However, there are also many other properties of surface reflectance, such as texture (spatial variation in how light is reflected), specularity, and translucence (the two latter are functions of the way light is scattered by the surface). Shading cues, discussed above, do not fit cleanly into the proposed distinction between shape and pigmentation. For pragmatic purposes in the current study, we will minimize shading cues and include them with shape cues in one experiment and with pigmentation cues in another. For the sake of brevity, surface reflectance properties have elsewhere been referred to as 'color' or 'texture'. Unfortunately these terms also have colloquial meanings that refer to subsets of surface reflectance properties (hue and spatial variation in reflectance, respectively). Thus we use the word 'pigmentation' for the sake of both brevity and clarity.

The evidence that the use of pigmentation is disrupted by negation derives from studies on uniformly pigmented 3-D face stimuli (Bruce and Langton 1994; Liu et al 1999). These images are of models with the 3-D shape of a particular face (as derived from laser scans) that reflect light uniformly from all points on the surface. Normal faces reflect light differently at different points across the face. The disruption in recognition performance caused by negation of these images of uniformly pigmented head models is much smaller than that caused by negation of normally pigmented faces. This difference has been interpreted as evidence that the perception of pigmentation cues to identity is what is normally disrupted by negation. However, it is also possible that there is no effect of negation with the uniformly pigmented faces because they are processed by the visual system in a fundamentally different way than normal faces. 
In particular, it has been proposed that the pattern of dark areas (corresponding to the eyes and lips) surrounded by lighter areas that is common to faces under normal lighting conditions underlies the process of face detection (Sinha 2002; Watt 1994). If this pattern of luminance is necessary for activating normal face processing, images without this pattern, such as images produced from the uniformly pigmented face models, may not receive the same processing as normal faces. For this reason, it would be desirable to determine whether pigmentation cues of faces with normal, face-like patterns of pigmentation are disrupted by negation.

For the present study we sought to determine whether shape (including second-order relations) or pigmentation is disrupted by negation. Our strategy was to create sets of faces that differed from one another in terms of only their shape or only their pigmentation, respectively the 'shape' and 'pigmentation' sets. However, unlike unpigmented face stimuli, the faces in both of these sets look like normal faces, with face-like shape and pigmentation. Subjects performed a delayed match-to-sample, two-alternative forced-choice task, in which they had to distinguish between two faces from either the shape or pigmentation set. In this way we controlled which cues were available for distinguishing the two faces. By comparing performance with shape and pigmentation cues in recognizing faces in positive or negative contrast, we could determine whether either or both cues are disrupted by negation. If the use of shape is disrupted by negation, performance with the shape set should have been significantly worse with negated faces than with positive faces. If the use of pigmentation is disrupted by negation, performance with the pigmentation set should have been significantly worse with negated faces than with positive faces.

\section{Methods}

We conducted two experiments that differed only in terms of the stimuli and the presentation timing. Both experiments employed full color stimuli. In the first experiment, we used artificially generated faces for which shape and pigmentation could be manipulated independently. To test whether the results of the first experiment generalize to real-world facial images, in the second experiment we used manipulated photographs of actual faces instead of artificial stimuli. These stimuli retained potentially relevant pigmentation cues such as specularity and translucence that are lost in 3-D models such as laser-scanned images or the artificial stimuli used in the first experiment. The stimuli in the two experiments differed in how shape and pigmentation were defined. For the artificial stimuli of the first experiment, shape was defined as the location of the face surface in 3-D, and pigmentation was defined as the way in which light reflected off the face surface. For the photographic stimuli of the second experiment, shape was defined as the locations of face contours in the 2-D image space, and pigmentation was defined as the pixel values at different locations of the image. A consequence of the different definitions of shape and pigmentation in the two experiments is that in experiment 1 , faces in the shape set differ in shading, while in experiment 2 , the pigmentation set contains the differences in shading. If the shading cues play a large role, we will expect different relative performances in the two experiments. However, we selected the illumination of the faces in both experiments to minimize shading differences between the faces (described below).

\subsection{Subjects}

A total of twenty-eight subjects participated in this study; fourteen in experiment 1 and fourteen in experiment 2. All were contacted through the MIT Brain and Cognitive Sciences subject pool, were naive to the purpose of the study, and had normal or corrected-to-normal vision. 


\subsection{Experimental design}

In both experiments we used the same delayed match-to-sample, two-alternative forcedchoice task, with the same experimental design (figure 1). In each trial of this task, the observer saw a fixation dot followed by a sample face, then a visual noise mask, then a blank screen. Next, two faces were presented side by side in the center of the screen. One of the two faces, the sample, had been presented just previously, and the other was a distractor face that differed from the target in shape only (shape condition), in pigmentation only (pigmentation condition), or in both shape and pigmentation (shape + pigmentation condition). The task was to decide which of the two faces matched the sample, and to press the corresponding key as quickly as possible. Trials from the different conditions were intermixed, and the left-right ordering of target and distractor was counterbalanced. There were eight male and eight female faces in each condition. All the faces in a trial were presented in positive contrast or in negative contrast. Each of the eight faces was paired with every other face of its same sex for each condition, resulting in 28 (pairs) $\times 2$ (sex) $\times 3$ (condition) $\times 2$ (contrast $) \times 2($ left - right counterbalance $)=672$ trials per observer. Stimuli were presented in Matlab (MathWorks) with the psychophysics toolbox extensions (Brainard 1997; Pelli 1997).

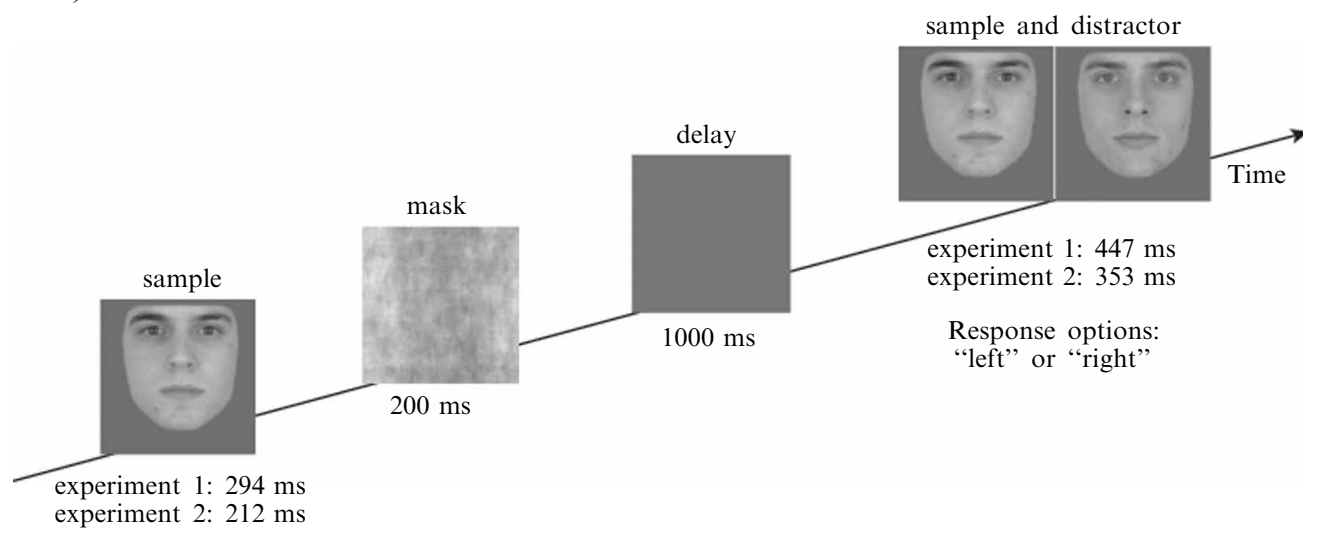

Figure 1. Experimental design. On each trial, subjects viewed a face, followed by a visual noise mask, then a delay. Two faces were then presented; one face (the target) was the identical image as the sample, and the other face (the distractor) was drawn from the same category of faces. For example, if the face in the upper left of figure 2 was the target, the face to the right could be the distractor, but not one of the faces below. In this way, the information distinguishing the target and distractor faces defined the condition: shape only, pigmentation only, and shape + pigmentation.

\subsection{Experiment 1: Stimuli and presentation}

Stimuli for the first experiment were produced with FaceGen Modeller 3.0 (Singular Inversions) software that creates 3-D models of artificial faces, with separate representations for shape and for pigmentation (referred to as 'texture' by the software). To make the faces appear as naturalistic as possible, we used 'photofits', in which frontal and profile photographs of actual faces determined the shape and pigmentation settings of the models. The photofit system is proprietary, and thus we do not know exactly how the 2-D photographic images were used to create separate pigmentation and 3-D shape. However, these details are irrelevant for the present purposes; the important point is that face representations could be created that varied in terms of both their 3-D shape and texture (pigmentation) models, and that these separate models could subsequently be altered independently. Photofits of eight male and eight female faces were created. The people on whom the photofits were based ranged in age from 18 to 25 years, with a mean age of 21 years. Because it would be less surprising if pigmentation were used to distinguish among faces of different races, 
all the faces were of the same race (Caucasian). The models generated directly from the photofits differed from one another in terms of both their shape and their pigmentation, and so they formed the stimuli for the shape + pigmentation condition. To create the stimuli for the pigmentation condition, the shape models for each of the photofit heads were given the same settings (the software's 20-year-old male or female Caucasian average), producing a set of faces each with distinct pigmentation, but the same (average) shape. To create the stimuli for the shape condition, the texture models for each of the photofit heads were given the same settings (the software's 20-year-old male or female Caucasian average), producing a set of faces, each with distinct shape, but the same (average) pigmentation. The models were rendered with a single light source at $0^{\circ}$, with the lighting source set to $60 \%$ with $40 \%$ ambient brightness. These lighting conditions were selected to minimize effects of shading cues, and were applied consistently in order to eliminate effects of varied lighting direction (Braje et al 1998; Moses et al 1994). A 7/8 viewpoint was selected to make both shape and pigmentation cues readily apparent. Examples of the stimuli can be seen in figure 2. Presentation times for experiment 1 were: probe $294 \mathrm{~ms}$, mask $200 \mathrm{~ms}$, delay $1000 \mathrm{~ms}$, target and distractor $447 \mathrm{~ms}$. These times were selected to elicit accuracy levels of approximately $75 \%$, in the center of the range between chance and perfect performance.

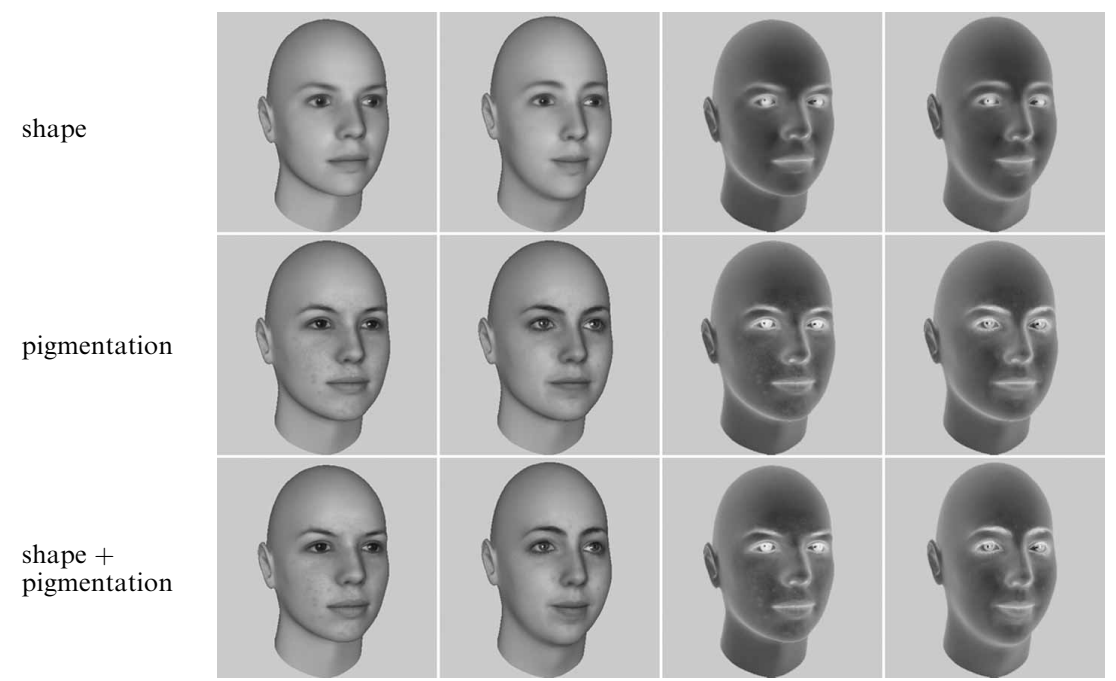

Figure 2. Examples of artificial stimuli. The shape-condition faces along the top rows of each set have the same pigmentation as one another and are distinguishable only by shape. The pigmentationcondition faces along the middle rows have the same shape and are distinguishable only by pigmentation. The shape + pigmentation faces along the bottom rows, like normal faces, are distinguishable by both shape and pigmentation. In both sets, the left two columns show faces with positive contrast, and the right two columns show the same faces with negative contrast. A color version of this figure can be viewed on the Perception website at http://www.perceptionweb.com/misc/p5490/.

\subsection{Experiment 2: Stimuli and presentation}

We took frontal photographs of eight male and eight female Caucasian faces, ranging in age from 18 to 25 years, with a mean age of 20 years. Large, diffuse lighting centered at $0^{\circ}$ elevation was used to eliminate cast shadows and to minimize the effects of shading cues. The heads of the models were placed in the same location relative to the lighting sources and the rest of the room, to ensure equivalent lighting the faces. These photographs were then manipulated with Morph Man 3.0 (Stoik Imaging). For each sex, the eight original faces were morphed together to produce an average face. We created the stimuli for the shape condition by warping (moving pixels in the image plane without averaging pixel values, as done in morphing) this average face 
into the shape of each of the original eight faces, producing eight new faces, each with the same (average) pigmentation, but with distinct shape. Similarly, we created the stimuli for the pigmentation condition by warping each of the eight original faces into the shape of the average face, which produced eight faces, each with the same (average) shape, but with distinct pigmentation. The original photographs of the eight faces of each sex, differing from one another in terms of both shape and pigmentation, formed the stimuli for the shape + pigmentation condition (see figure 3). Presentation times for experiment 2 were: probe $212 \mathrm{~ms}$, mask $200 \mathrm{~ms}$, delay $1000 \mathrm{~ms}$, target and distractor $353 \mathrm{~ms}$. The probe and target were presented more briefly in experiment 2 than in experiment 1 in order to maintain accuracy levels around $75 \%$.

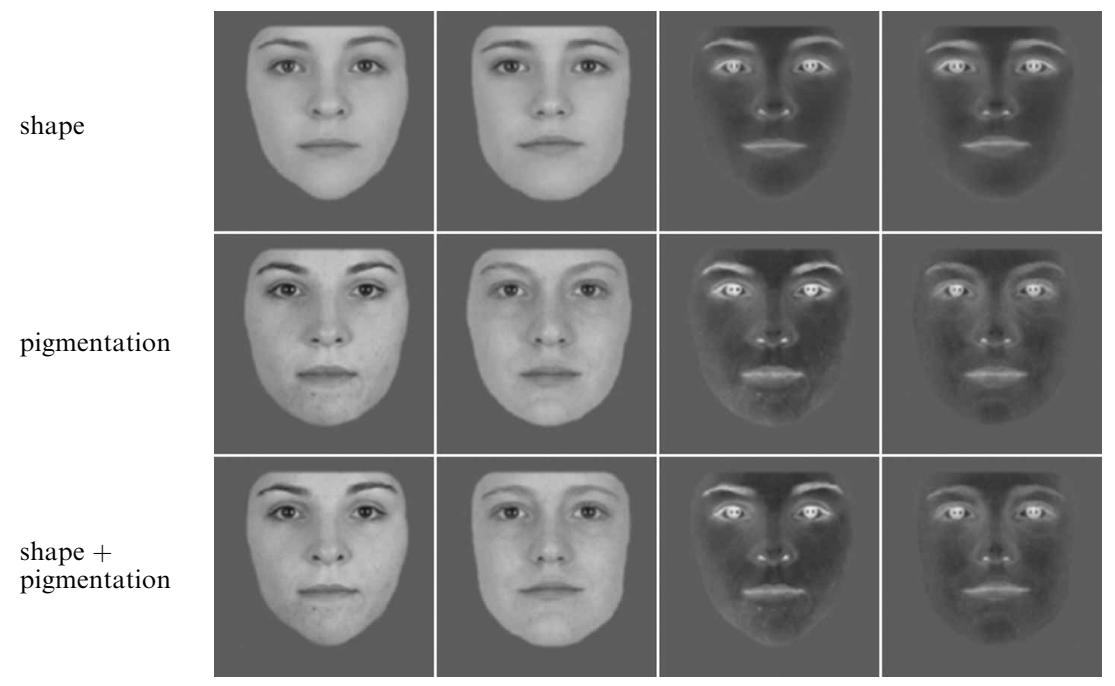

Figure 3. Examples of photographic stimuli. The faces are arrayed in the same way as in figure 2 . A color version can be viewed on the Perception website at http://www.perceptionweb.com/misc/p5490/.

\section{Results}

\subsection{Experiment 1}

The first experiment used artificial stimuli (figure 2 and section 2.3). We recorded observers' performance on a delayed match-to-sample, two-alternative forced-choice task (figure 1 and section 2.2). Figure 4 shows that negation impaired performance (producing higher error rates) in the pigmentation and shape + pigmentation conditions, but not the shape condition. That is, when only shape cues were available for the discrimination of the faces, performance was not significantly worse with negated faces than positive faces. There was a main effect of condition $\left(F_{2,26}=14.1, p<0.001\right)$, with the best performance when both shape and pigmentation cues were available. There was also a main effect of contrast $\left(F_{1,13}=62.2, p<0.001\right)$, with performance worse on negative than positive faces, consistent with all other studies on the effect of negation. Almost all this cost of negation was on pigmentation cues, producing a significant interaction between condition and contrast $\left(F_{2,16}=3.7, p<0.05\right)$. A posteriori comparisons of performance on positive and negative contrast faces within each condition showed significant differences ( $p<0.05$, Bonferroni corrected) between the positive and negative versions only for the two conditions with pigmentation cues (pigmentation and shape + pigmentation), but not for the shape condition.

These were not floor or ceiling effects in that the shape condition, which showed no effect of negation, was midway between the pigmentation and shape + pigmentation conditions. 


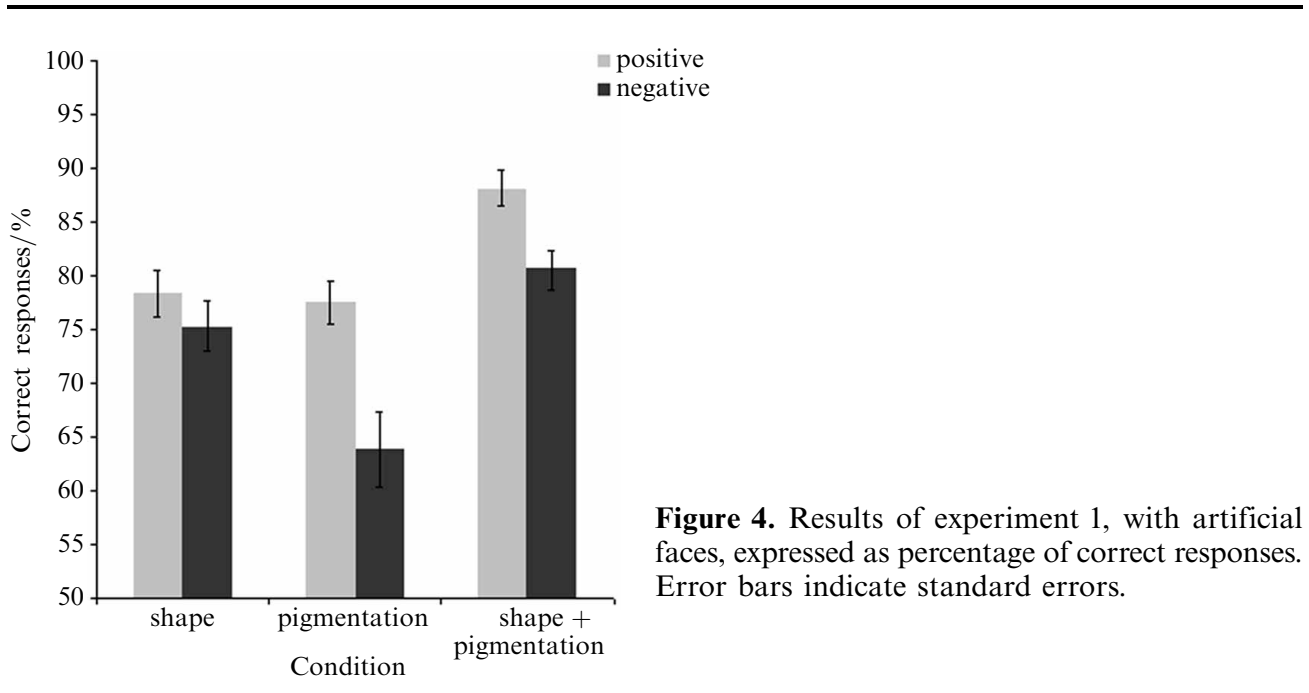

\subsection{Experiment 2}

Because the artificial stimuli in experiment 1 were not entirely naturalistic in appearance, the second experiment used stimuli created from photographs of actual faces (figure 3 and section 2.4) to assess whether the results of experiment 1 do indeed generalize to more naturalistic face images. The same task and experimental design were used as in experiment 1 (figure 2 and section 2.2). The pattern of results from experiment 2 was the same as that from the first experiment (figure 5). There were main effects of condition $\left(F_{2,26}=25.1, p<0.001\right)$, with performance best in the shape + pigmentation condition; and of contrast $\left(F_{1,13}=30.5, p<0.001\right)$, with performance better on positive than negative faces. There was also a significant interaction between condition and contrast $(F=5.0, p<0.05)$, and a posteriori comparisons of performance on positive and negative contrast faces within each condition showed significant differences $(p<0.05$, Bonferroni corrected) between positive and negative conditions only for the two conditions with pigmentation cues (pigmentation and shape + pigmentation), but not for the shape condition.

Data from a more naturalistic set of photographic stimuli thus provide further evidence that faces are difficult to recognize in contrast negative because pigmentation cues are rendered unusable. An alternative hypothesis, that perception of second-order relations is disrupted by negation, was not supported.

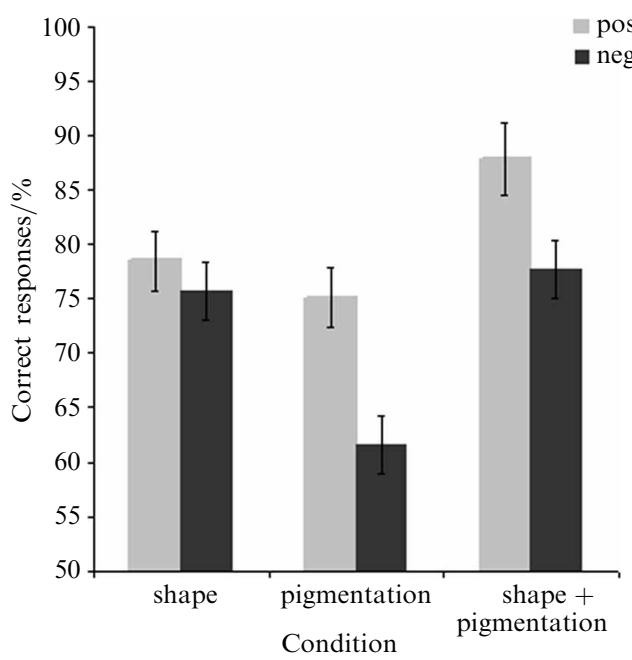

Figure 5. Results of experiment 2, with photographic faces, expressed as percentage of correct responses. Error bars indicate standard errors. 


\section{Discussion}

Why is it difficult to recognize faces in photographic negative? In two experiments, face recognition was significantly disrupted by negation when pigmentation cues provided the discriminable information, but when only shape cues were discriminable, negation had no significant effect. These results are consistent with the notion that negation disrupts our ability to use pigmentation cues. Because negation severely disrupts our ability to recognize faces, an implication of this finding is that pigmentation is one of the main components of the representation of face identity. Our results are consistent with those from other studies finding a role for pigmentation in face recognition (Bruce and Langton 1994; O’Toole et al 1999), as well as facial sex classification (Hill et al 1995; Tarr et al 2001) and attractiveness (Jones et al 2004; Russell 2003). Additional work from our labs suggests that the patterns of pigmentation around the eye regions play a particularly important role in judgments of identity (Sinha and Gilad 2004).

The findings also provide evidence against an alternative hypothesis why negation impairs recognition performance: that the perception of second-order relations of features is disrupted by negation. In each of the two experiments, there was no significant effect of negation when only shape cues were available. Discontinuous contours, such as line drawings and high-pass filtered images, are not disrupted by negation (Hayes et al 1986). Only the faces in the shape condition differed in terms of discontinuous contours, and it is possible that subjects adopted a strategy in the shape condition of focusing only on discontinuous contours, for example the outline of the face. Two lines of evidence argue against this possibility. First, in our experiments we used randomized rather than blocked presentation, such that subjects could not anticipate which cues would be useful for subsequent matching. Second, while there are readily distinguishable differences in the discontinuous contours of the photographic stimuli of experiment 2, there are not such readily distinguishable differences among the artificial faces of experiment 1 (compare figures 2 and 3).

This finding that the perception of shape cues, including second-order relations of features, is not disrupted by negation is somewhat at odds with two previous studies. Kemp et al (1990) found that, when viewing negative images, subjects were less sensitive to changes to the distances between features, which would suggest that negation disrupts second-order relations. However, the stimuli for those experiments consisted of two-tone (black or white) images of a single face. In a subsequent study, Liu and Chaudhuri (1997) found that performance on an old-new task (without manipulation of distances between features) was more impaired by negation with two-tone than continuous tone images. This suggests that the Kemp et al (1990) finding may have been an artifact of the stimulus type rather than the manipulation of second-order relations. Lewis and Johnston (1997) reported that, with faces presented in negative contrast, subjects were slower at determining whether a pair of faces differed in terms of being 'thatcherised' or not. This finding was interpreted as evidence that negation disrupts the perception of second-order relations ('configuration' in their terminology). Given that thatcherisation does not change the second-order relations of a face, it is not clear why the slower performance with negated faces should be interpreted in this way. We believe that the present findings constitute a more direct test of the hypothesis that shape (including second-order relations) is disrupted by negation.

Because negation is so detrimental to recognition performance, the observation that negation specifically disrupts pigmentation cues implies that pigmentation is an important component of identity for normal recognition (ie with positive contrast). However, negation is not the sole litmus test of importance for recognition, and so the results do not imply that shape is not also an important component of identity for normal recognition. In addition to evidence from many other studies, two findings from the present investigation suggest that shape is indeed important. The first is that 
performance with positive contrast in the shape condition was well above chance, which indicates that shape alone can be used to recognize faces. The second is that performance with negative contrast in the shape + pigmentation condition was also well above chance, illustrating that faces can still be recognized after negation. Indeed, performance with negative contrast in the shape + pigmentation condition was almost the same as performance with positive contrast in the shape condition, consistent with the idea that negation selectively disrupted pigmentation cues, leaving shape cues unaffected.

We have used a task that involved the matching of unfamiliar faces, and so we cannot be certain that these results would generalize to recognition memory for familiar faces. However, several reasons suggest that such generalization is likely. In many studies of face recognition, including a majority of those cited here, use has been made of face matching rather than familiar recognition tasks, and most problems associated with familiar recognition, such as inversion and negation, are also found with face matching, suggesting important commonalities between matching and recognition. Our task also had a memory component-subjects performed the matching task after viewing a visual noise mask and more than a second of delay-indicating that perceptual comparison alone was not sufficient for performance. In any case, matching unfamiliar faces is an important task worth investigating in its own right (Hancock et al 2000), for its application to eyewitness testimony and for the construction of automatic systems of face recognition.

How specific are these findings to faces? Negation has been found not to disrupt subordinate-level recognition of at least one class of object-chairs (Subramaniam and Biederman 1997), and of artificial 'blobs' [asymmetrical harmonics of a sphere with smooth surfaces (Nederhouser et al 2003)]. Material perception, however, is disrupted by negation (Fleming et al 2001, 2004). In the negated images of figure 6, as an example, we can see what kinds of objects are represented, but it is very difficult to recognize the materials of which they are composed. This difference in susceptibility to negation is likely due to the different kinds of information available for recognition of objects and materials. There is agreement that shape is the primary cue for recognition of non-face objects - generally referred to with 'count nouns', such as horse, house, and hammer - and that pigmentation usually plays a secondary role (Biederman and $\mathrm{Ju}$ 1988; Tanaka et al 2001). In contrast, materials-commonly referred to with 'mass nouns', such as wax, wood, and water-are recognized almost entirely on the basis of their pigmentation (surface reflectance properties) (Biederman 1987). This bolsters the argument that negation disrupts the use of pigmentation cues, and suggests that the phenomenon may not be specific to faces, but rather a general property of recognition.

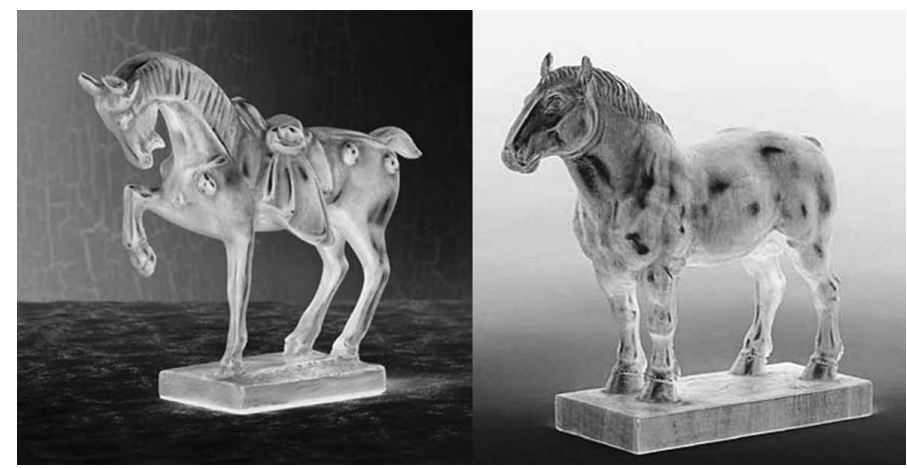

Figure 6. Two negative-contrast images. It is readily apparent that both images depict statues of horses. However, it is much more difficult to determine that the statue on the left is constructed of bronze, and the statue on the right is of wood. In positive contrast, recognizing these materials is easy. 
Although face recognition is typically compared with object recognition, these findings suggest that another fruitful comparison would be to material perception, which also depends critically on surface reflectance properties.

For humans, the task of recognizing faces is both difficult and extremely important. It would not make sense for our recognition system to throw away useful information. We have presented evidence from contrast negation that pigmentation is an important part of face identity. This finding cautions against the use of stimuli for recognition experiments that do not retain pigmentation cues, and is not consistent with models of face recognition that throw away pigmentation cues to rely entirely on facial-metric cues such as the shape, size, or distance between features.

Acknowledgments. We thank Philip Matthew Bronstad, Jodi Davenport, Ken Nakayama, and Mary Potter for comments on earlier versions of the manuscript, and to the members of the Sinha and Biederman labs for their helpful comments and technical assistance. This research was supported by a grant to PS from the DARPA Human ID Program administered by the Information Awareness Office, and by grants ARO DAAG55-98-1-0293, NSF EEC-9529152, HFSP 99-44, JSMF No. 96-44 to IB. RR was supported by an NIH training grant.

\section{References}

Biederman I, 1987 "Recognition-by-components: a theory of human image understanding" Psychological Review 94115 - 147

Biederman I, Ju G, 1988 "Surface versus edge-based determinants of visual recognition" Cognitive Psychology $2038-64$

Brainard D H, 1997 "The psychophysics toolbox" Spatial Vision $10433-436$

Braje W L, Kersten D, Tarr M J, Troje N F, 1998 "Illumination effects in face recognition" Psychobiology $26371-380$

Bruce V, Langton S, 1994 "The use of pigmentation and shading information in recognising the sex and identities of faces" Perception $23803-822$

Bruce V, Young A, 1998 In the Eye of the Beholder: The Science of Face Perception (Oxford: Oxford University Press)

Diamond R, Carey S, 1986 "Why faces are and are not special: an effect of expertise" Journal of Experimental Psychology: General $115107-117$

Fleming R W, Dror R O, Adelson E H, 2001 "How do humans determine reflectance properties under unknown illumination?", in CVPR (Kauai, Hawaii: IEEE)

Fleming R W, Jensen H W, Bülthoff H H, 2004 "Perceiving translucent materials", in $A C M$ SIGGRAPH (Los Angeles, CA: Association for Computing Machinery)

Freire A, Lee K, Symons L A, 2000 "The face-inversion effect as a deficit in the encoding of configural information: Direct evidence" Perception 29 159-170

Galper R E, 1970 "Recognition of faces in photographic negative" Psychonomic Science 19 207-208

Galper R E, Hochberg J, 1971 "Recognition memory for photographs of faces" American Journal of Psychology $84351-354$

George N, Dolan R J, Fink G R, Baylis G C, Russell C, Driver J, 1999 "Contrast polarity and face recognition in the human fusiform gyrus" Nature Neuroscience 2 574-580

Hancock P J B, Bruce V, Burton A M, 2000 "Recognition of unfamiliar faces" Trends in Cognitive Sciences $4330-337$

Hayes T, Morrone M C, Burr D C, 1986 "Recognition of positive and negative bandpass-filtered images" Perception $15595-602$

Hill H, Bruce V, 1996 "Effects of lighting on the perception of facial surfaces" Journal of Experimental Psychology: Human Perception and Performance 22 986-1004

Hill H, Bruce V, Akamatsu S, 1995 "Perceiving the sex and race of faces: the role of shape and colour" Proceedings of the Royal Society of London B 261 367-373

Hole G J, George P A, Dunsmore V, 1999 "Evidence for holistic processing of faces viewed as photographic negatives" Perception 28341 - 359

Horn B K H, 1986 Robot Vision (Cambridge, MA: MIT Press)

Johnston A, Hill H, Carman N, 1992 "Recognising faces: effects of lighting direction, inversion, and brightness reversal" Perception $21365-375$

Johnston A, Passmore P J, 1994 "Shape from shading. I: Surface curvature and orientation" Perception $23169-189$

Jones B C, Little A C, Burt D M, Perrett D I, 2004 "When facial attractiveness is only skin deep" Perception 33 569-576 
Kemp R, McManus C, Pigott T, 1990 "Sensitivity to the displacement of facial features in negative and inverted images" Perception $19531-543$

Kemp R, Pike G, White P, Musselman A, 1996 "Perception and recognition of normal and negative faces: the role of shape from shading and pigmentation cues" Perception $2537-52$

Leder H, Bruce V, 2000 "When inverted faces are recognized: the role of configural information in face recognition" Quarterly Journal of Experimental Psychology A: Human Experimental Psychology $53513-536$

Leder H, Candrian G, Huber O, Bruce V, 2001 "Configural features in the context of upright and inverted faces" Perception $3073-83$

LeGrand R, Mondloch C J, Maurer D, Brent H P, 2001 "Early visual experience and face processing" Nature 410890

Lewis M B, Johnston R A, 1997 "The Thatcher illusion as a test of configural disruption" Perception $26225-227$

Liu C H, Chaudhuri A, 1997 "Face recognition with multi-tone and two-tone photographic negatives" Perception $261289-1296$

Liu C H, Collin C A, Burton A M, Chaudhuri A, 1999 "Lighting direction affects recognition of untextured faces in photographic positive and negative" Vision Research $394003-4009$

Liu C H, Collin C A, Chaudhuri A, 2000 "Does face recognition rely on encoding of 3-D surface? Examining the role of shape-from-shading and shape-from-stereo" Perception 29 729-743

Moses Y, Adini Y, Ullman S, 1994 "Face recognition: the problem of compensating for illumination changes" Proceedings of the European Conference on Computer Vision (Berlin: Springer) pp $286-296$

Nederhouser M, Mangini M C, Biederman I, Okada K, 2003 "Invariance to contrast inversion when matching objects with face-like surface structure and pigmentation" Journal of Vision 393

O'Toole A J, Vetter T, Blanz V, 1999 "Three-dimensional shape and two-dimensional surface reflectance contributions to face recognition: an application of three-dimensional morphing" Vision Research $393145-3155$

Pelli D G, 1997 "The VideoToolbox software for visual psychophysics: Transforming numbers into movies" Spatial Vision $10437-442$

Phillips R J, 1972 "Why are faces hard to recognize in photographic negative" Perception \& Psychophysics $12425-426$

Ramachandran V S, 1988 "Perception of shape from shading" Nature 331 163-166

Russell R, 2003 "Sex, beauty, and the relative luminance of facial features" Perception 321093 - 1107

Sinha P, 2002 "Qualitative representations for recognition" Lecture Notes in Computer Science $2525249-262$

Sinha P, Gilad S, 2004, "Face recognition with contrast chimeras" Journal of Vision 4216 (abstract)

Subramaniam S, Biederman I, 1997 "Does contrast reversal affect object identification" Investigative Ophthalmology \& Visual Science 38998

Tanaka J, Weiskopf D, Williams P, 2001 "The role of color in high-level vision" Trends in Cognitive Sciences $5211-215$

Tarr M J, Kersten D, Cheng Y, Rossion B, 2001 "It's Pat! Sexing faces using only red and green" Journal of Vision 1337

Watt R J, 1994 "A computational examination of image segmentation and the initial stages of human vision" Perception $23383-398$

White M, 2001 "Effect of photographic negation on matching the expressions and identities of faces" Perception $30969-981$ 


\section{PERCEPTION}

VOLUME 352006

www.perceptionweb.com

Conditions of use. This article may be downloaded from the Perception website for personal research by members of subscribing organisations. Authors are entitled to distribute their own article (in printed form or by e-mail) to up to 50 people. This PDF may not be placed on any website (or other online distribution system) without permission of the publisher. 